REVISTA DE DERECHO UNED, núm. 3, 2008

\title{
PRINCIPALES RECURSOS DE INFORMACIÓN PARA LA FILOSOFÍA JURÍDICA: LAS GUÍAS DE FUENTES
}

\author{
ISABEL VILLASEÑOR RODRÍGUEZ \\ Profesora Titular de la Facultad de Ciencias de la Documentación. \\ Universidad Complutense de Madrid
}

Resumen: El texto presenta una selección de fuentes de información de gran utilidad para iniciar la búsqueda de información en Filosofía jurídica y disciplinas afines. La necesidad de controlar la producción bibliográfica actual impone el manejo de recursos que recojan aquellas fuentes de interés para acceder a la información que necesitamos. En el presente trabajo se ofrecen algunas referencias que permitirán el conocimiento de esas fuentes, así como una breve caracterización con el fin de facilitar su uso. Se ofrece, pues, un repertorio de guías de fuentes de información que pueda servir como herramienta que oriente en el conocimiento y manejo de aquellos recursos informativos útiles para los usuarios que deseen conocer esta información: estudiantes, profesores, investigadores y profesionales de la información, iniciados o no en estas disciplinas.

Palabras clave: Documentación iusfilosófica. Fuentes de información. Guías de fuentes de información especializadas.

Abstract: The text presents a selection of sources of information useful to start searching for information on legal philosophy and related disciplines. The need to control the output current literature imposes resource management which collect those sources of interest to access the information they need. In this paper are some references that allow the knowledge from these sources, as well as a brief characterization in order to facilitate its use. It offers a directory of guides to in- 
formation sources that can serve as a tool to guide on knowledge and management of information resources useful for those users who want to know this information: students, teachers, researchers and information professionals, initiated or not in these disciplines.

Sumario. I. Introducción. II. Algunas guías de fuentes de información. II. 1. Guías generales. II. 2. Guías especializadas en Humanidades y Ciencias Sociales. II. 3. Guías especializadas en Filosofía. II. 4. Guías especializadas en Derecho y Filosofía jurídica

\section{INTRODUCCIÓN}

Ante la ingente cantidad de información producida y, lo que es más alarmante, la creciente dificultad para acceder a la que realmente nos interesa, la existencia de recursos que den cuenta de todas aquellas fuentes de interés para poder encontrarla es, cada día, más necesaria. Toda la información disponible no es aprovechable si no se utiliza, y no todos los implicados en una determinada disciplina conocen todas las fuentes de información posibles, e incluso, si las conocieran, no siempre las utilizarían correctamente por falta de adiestramiento en las técnicas documentales de recuperación de la información. De ahí que empiecen a proliferar publicaciones que pretenden dar cuenta de aquellos recursos de interés para los distintos sectores de la actividad humana. Cada uno de ellos ofrece su visión personal del asunto, ofreciendo criterios particulares a la hora de seleccionar, organizar, e incluso describir, quizá porque no exista un referente adecuado que permita desarrollar una metodología más o menos uniforme.

Este tipo de publicaciones han dado en llamarse, en el ámbito de la Documentación, de muy diversas maneras. En inglés encontramos los términos general works ${ }^{1}$, access to sources ${ }^{2}$, reference books and research guides ${ }^{3}$, guide to refence material ${ }^{4}$; en francés, guides du biblio-

1 GUIDE to reference books, edit. by Eugene P. Sheehy, with the assistance of Rita G. Keeckeissen,10th. ed., Chicago, American Library Association, 1986.

${ }^{2}$ LI, T.Ch., Social science reference sources: a practical guide, 3ed. ed., Westport (Connecticut), London, Greenwood Press, 2000.

3 BLAZEK, R., AVERSA, E., The Humanities: a selective guide to information sources, 4th. ed., Englewood, Libraries Unlimited, 1994.

4 WALFORD's guide to reference materials, 7th. ed., London, Library Association, 1996-1998, $3 \mathrm{v}$. 
thecaire ${ }^{5}$; en español, obras de consulta sobre obras de consulta ${ }^{6}$, guía de obras de referencia y consulta ${ }^{7}$, directorio de recursos ${ }^{8}$, repertorios de obras de referencia ${ }^{9}$ y fuentes secundarias reelaboradas ${ }^{10}$, por ejemplo. También varía de unos autores a otros la opinión respecto a qué tipo de recursos puedan considerarse dentro de estos parámetros ${ }^{11}$.

En el presente trabajo hemos preferido utilizar el término "guía de fuentes de información» para referirnos a este tipo de obras, teniendo en cuenta su carácter eminentemente introductorio e instrumental. Entendemos por tal, aquel documento que, de una manera u otra, nos aporta información sobre todos aquellos recursos de interés para encontrar la información que buscamos. Permite iniciar el proceso de búsqueda y debe entenderse como el primer estadio del mismo. Son fuentes documentales de carácter terciario en tanto que dan información de otras fuentes (las secundarias) que serán las que nos lleven a la información que necesitemos.

En cuanto a los documentos que pueden considerarse como «guías de fuentes», se puede decir que son de tres tipos:

Las guías de obras de referencia

Los manuales de fuentes de información

Las bibliografías de bibliografías

5 BEAUDIQUEZ, M., Guide de bibliographie générale. Méthodologie et pratique, nouvelle ed. rev. et mise à jour, München [etc.], Saur, 1989.

6 GARZA MERCADO, A., Obras de consulta para estudiantes de Ciencias Sociales y Humanidades, México, El Colegio de México, Biblioteca Daniel Cosío Villegas, 1992; Obras generales de consulta, México, Biblioteca Daniel Cosío Villegas, El Colegio de México, 1997.

7 LÓPEZ CASTRO, M. M., JIMÉNEZ PELAYO, J., Fuentes de información sobre Andalucía: Guía de obras de referencia y consulta, (s.l.), Junta de Andalucía, Consejería de Cultura, 2001.

${ }^{8}$ La INFORMACIÓN especializada en Internet: directorio de recursos de interés académico y profesional, coordinación de Ángeles Maldonado Martínez y Luis Rodríguez Yunta, 2. ${ }^{a}$ ed. corr. y aum., Madrid, CSIC, 2006.

9 AYUSO GARCÍA, M. D., «Revisión interdisciplinar de las fuentes de información en los umbrales del siglo xxI. Nuevas perspectivas: los recursos de información», en Revista General de Información y Documentación, vol. 9, n. ${ }^{\circ} 1,1999$, págs. 203-215.

10 MARTÍN VEGA, A., Fuentes de información general, Gijón, Trea, 1995.

11 Sheehy considera que son las guías, los manuales de fuentes de información y las bibliografías de bibliografías; S. Romanos de Tiratel (Guía de fuentes de información especializadas. Humanidades y Ciencias Sociales, Buenos Aires, EB, 1996 y Guía de fuentes de información especializadas: Humanidades y Ciencias Sociales, $2 .^{\mathrm{a}}$ ed., Buenos Aires, GREBYD, 2000) que son las guías de obras de referencia y las bibliografías de bibliografías; Li, las guías y las bibliografías de bibliografías; y Garza, solamente estas últimas. 
Las guías de obras de referencia

Dado el enorme crecimiento experimentado en los últimos años por la literatura secundaria, es decir, por todo tipo de bibliografías y obras de referencia, la necesidad de poderlas conocer ha hecho que surjan otras fuentes que remiten a ellas y que se han dado en llamar fuentes de carácter terciario. El interés de estas fuentes reside en que constituyen el primer paso en cualquier proceso de investigación y búsqueda de información, ya que proporcionan el conocimiento de aquellas fuentes que a su vez van a conducir al documento originario que sea de interés. A estas fuentes de carácter terciario se las viene llamando guias de obras de referencia o guias bibliográficas, y son de muy distintas características. Fundamentalmente, conviene distinguir entre las que abarcan todas las materias (generales) y las que se limitan a un área disciplinaria o a una sola materia (especializadas). También están las que ofrecen una relación de esas fuentes secundarias sin más y las que incluyen juicios valorativos de cada una de ellas, o se limitan a un territorio y o a una época determinados. Se crean para orientar al investigador en un campo del saber o para aconsejar a los profesionales de la información sobre los recursos con los que deben contar en sus centros. Identifican, enumeran y describen fuentes secundarias. Suelen introducir al lector en el campo temático y sus subtemas, seleccionando, guiándole en las diferentes partes de una disciplina y enseñándole cómo buscar información sobre una materia, cómo manejar las fuentes.

Internet permite hoy en día la posibilidad de contar con algunas de estas guías de fuentes que, en algunas ocasiones, han dado en llamarse guías de recursos de información. Las ventajas sobre las aparecidas en papel son evidentes: su actualidad, su rápida actualización y el gran número de recursos que ofrecen. Las hay generales y especializadas ${ }^{12}$, elaboradas por entidades públicas (bibliotecas, por ejemplo $^{13}$ ) o privadas (revistas telemáticas ${ }^{14}$, páginas personales), que recogen un tipo de fuentes (electrónicas) o todas; locales (recogen los recursos de su propiedad o ubicados en su centro) o mixtas (recogen recursos que existan en general). Son un compendio de recursos que se completan con lo que, en algunas páginas del tipo que sea, reciben el nombre de enlaces.

${ }^{12}$ Un ejemplo puede ser el de «Recursos de Información para Derecho en la Biblioteca», de la Universidad Carlos III.

${ }^{13}$ La mayoría de las bibliotecas especializadas españolas ofrecen este servicio. Véase cualquiera de las páginas de las distintas bibliotecas de la Universidad Complutense, por ejemplo.

${ }^{14}$ La Revista Telemática de Filosofía del Derecho, por ejemplo. 


\section{Los manuales de fuentes de información}

Por otra parte, el desarrollo de la investigación en Ciencia documental ha hecho que surjan publicaciones que, con un afán introductorio, ofrecen al lector consejos y recursos necesarios para iniciarse en la búsqueda de información, explicando los principios y procedimientos básicos de esa búsqueda, el manejo de los mismos y registrando las fuentes básicas.

En relación con este asunto está el de la proliferación de manuales creados como apoyo a la formación de futuros profesionales de la información. De entre ellos conviene destacar los surgidos para explicar qué se entiende por referencia o por fuente de información como herramienta imprescindible para llevar a cabo dicha tarea, cuáles son las fuentes de mayor interés para realizar esa labor profesional, cómo se estructuran y deben tratarse, qué criterios deben tenerse en cuenta ante la elección de una o de otra y ejemplos de cada uno de los tipos. En este caso, el problema fundamental que plantean es el de la necesidad de actualización, que no es habitual que se considere.

\section{Las bibliografías de bibliografias}

Son repertorios que recogen y describen un solo tipo de obras de referencia: las bibliografías. Su cobertura temática puede ser general o especializada, y su cobertura geográfica distinta. Pueden estar ordenados de distintas maneras y presentarse en distintos soportes. Se ofrecen periódicamente o no. Son una herramienta esencial en la búsqueda de información porque permiten conocer los repertorios bibliográficos que se han compilado sobre los temas de interés.

\section{ALGUNAS GUÍAS DE FUENTES DE INFORMACIÓN}

Por todo lo dicho anteriormente incluimos a continuación una breve relación de aquellos recursos de carácter terciario, en papel o soporte informático, que pueden servir para encontrar referencias de interés para el tema que nos ocupa. La única distinción que se ha hecho es entre guías de carácter general y guías de carácter especializado, individualizando las que se conocen sobre materias afines o relacionadas con el tema de estudio y ordenándolas alfabéticamente por el nombre del autor o el título. Aunque la mayoría son guías de fuentes españolas, se incluyen referencias en otras lenguas distintas 
de la española con el fin de completar las lagunas que existen en ella en relación con este asunto. Junto a las referencias bibliográficas, se incluye un breve resumen de contenido con el fin de poder guiar en su selección y manejo. Tan sólo en el caso de fuentes que presentan características muy similares no se ha incluido ese breve resumen por no ser reiterativos; es el caso de las páginas de bibliotecas universitarias. En cuanto a la cobertura cronológica, el presente trabajo contiene referencias de fuentes publicadas desde el siglo XIX hasta la actualidad.

Es evidente que no se pretende, ni sería posible, dar cuenta de la totalidad de las fuentes, ser omnicomprensivos. Se ofrece una selección de los recursos que se consideran más significativos, de referencia fundamental no sólo para quien quiera iniciarse, sino también para los especialistas y los profesionales de la información que deban tratar con una disciplina de naturaleza problemática. Así pues, las fuentes que se ofrecen se han seleccionado según su utilidad e idoneidad, y según su representatividad e importancia relativa.

\section{II.1. GUÍAS GENERALES}

- BEAUDIQUEZ, Marcelle: Guide de bibliographie générale. Méthodologie et pratique. Nouv. éd. rev. et mise à jour. München, London, New York, Paris: Saur, 1989

Imprescindible manual que estudia los distintos tipos de fuentes de información documentales, ofreciendo una tipología de gran interés, así como completos cuadros sobre la valoración de estas fuentes y ejemplos que, aunque en francés mayoritariamente, se complementan con obras de otras lenguas.

- BELTRÁN Y DE TORRES, Francisco: Biblioteca bio-bibliográfica: Catálogo de una importante colección de libros y folletos españoles y extranjeros referentes a bibliografía, biografía, bibliofilia, la imprenta y sus artes auxiliares, formada, catalogada y puesta en venta por Francisco Beltrán, librero-editor. Madrid: Librería Española y Extranjera, 1927

Aunque se trata de un catálogo comercial y de principios del siglo xx aún hoy en día mantiene su interés por la información contenida sobre esa época.

- BESTERMAN, Theodore: A World Bibliography of Bibliographies and bibliographical Catalogues, Calendars, Abstracts, Di- 
gest, Indexes and the Like... 4th. ed. Lausanne: Societas Bibliographica, 1965-1966. $5 \mathrm{v}$.

Incluye bibliografías de todas las áreas del conocimiento, colecciones de sumarios, resúmenes, compilaciones, catálogos comerciales, catálogos de bibliotecas, etc. Dividida por materias y dentro de cada una de ellas se subdividen las bibliografías según la fecha de publicación y número de referencia. Obra «monumental» cuya consulta es obligada. Cuenta con un suplemento publicado por TOOMEY.

- BIBLIOGRAPHIC index: a cumulative bibliography of bibliographies. 1937/1942- . New York: Wilson, 1945- .

Sólo registra bibliografías cerradas e intenta ser exhaustiva; ordena sus entradas bajo encabezamientos de materias e incluye las bibliografías aparecidas como libros, en libros y en unas 2600 publicaciones periódicas. Tiene acumulaciones anuales. Se puede consultar en CD ROM y en línea.

- BIBLIOGRAPHICAL Index: A Cumulative Bibliography of Bibliographies. New York: The Wilson Co., 1937-1949

Publicación trimestral que recoge bibliografías aparecidas en libros de reciente aparición. Complemento de la obra de Bestermann.

- BIBLIOTECA Bibliográfica Hispánica. Madrid: Fundación Universitaria Española, 1975-1987. 6 v.

Cada volumen recoge la información ordenada según distintos criterios: lugar de nacimiento y profesión de los autores, lugar de publicación de las bibliografías, órdenes religiosas...

- CARRIZO SAINERO, Gloria; Irureta-Goyena Sánchez, Pilar; López de Quintana Sáenz, Eugenio: Manual de fuentes de información. Zaragoza: CEGAL, 2000

Aunque se trata de un manual de fuentes de información, recoge entre sus ejemplos, algunas fuentes de carácter general que sirven de guía en la búsqueda de información.

- COLEX Data (http://www.colex-data.com)

Ofrece enlaces a sitios de interés jurídico, actualizados continuamente, tales como publicaciones y acceso a instituciones de todo tipo.

- COMPLURED. Biblioteca de la Universidad Complutense (http://www.ucm.es/bucm/complured.htm) 
CompluRed es un servicio gratuito de acceso a recursos de información multidisciplinares, accesibles en Internet: periódicos y revistas electrónicas, catálogos de bibliotecas, bases de datos, obras de referencia, asociaciones profesiones, foros de discusión, estadísticas, legislación, libros electrónicos, mapas, tesis, patentes y normas, etc. La interfaz de acceso al servicio permite al usuario hacer consultas y utilizar un sistema de navegación, sustentado por una clasificación de materias generales y sub-materias, hasta llegar a los recursos web. Además, permite buscar y limitar las búsquedas por algunos criterios como la lengua, el tipo de recurso, si es un recurso de la Universidad Complutense o tiene texto completo. Un aspecto singular de este servicio es que los recursos web que componen la base de datos han sido seleccionados por los bibliotecarios de la Universidad Complutense, con el objeto de apoyar a alumnos, profesores e investigadores en el aprendizaje, enseñanza e investigación.

- ENCICLOPEDIA de orientación bibliográfica. Edit. Por Tomás Zamarriego. Barcelona: Flors, 1964-65. 4 v.

Incluye bibliografías generales, divididas por materias y por tomos, con indicaciones sobre su uso.

- ENCICLOPEDIAS.com (http://www.enciclopedias.com)

Buscador de enciclopedias en Internet. Permite la búsqueda en inglés, francés y español.

- Las FUENTES de Información. Estudios teórico-prácticos. Edit. Por Isabel de Torres Ramírez. Madrid: Síntesis, 1998

Completo manual de fuentes de información que aporta, además de todo tipo de fuentes, referencias esenciales para los pasos iniciales de toda búsqueda informativa.

- FUENTES DE información en línea (http://ebro3.unizar.es:8080/bdl)

Directorio internacional de bases de datos elaborado por la Universidad de Salamanca.

- GALE Directory of Databases. Detroit: Gale, 1998

Recoge información de bases de datos de todas las materias y en distintos soportes. Cada entrada describe contenidos, informa sobre productores y vendedores así como sobre el idioma y la frecuencia de actualización. 
- GALE guide to Internet database. Edit. by Joanna Zakalik. New York: Gale Research, 1995

De ordenación alfabética, cuenta con índices de organismos, bases de datos, personas y materias.

- GUIDE to reference books, edit. by Eugene P. Sheehy; with the assistance of Rita G. Keeckeissen.10 th. ed. Chicago: American Library Association, 1986

-: covering materials from 1985-1990. Supplement to the 10 th. ed.; edited by Robert Balay. Chicago: American Library Association, 1992

Clásica guía de obras de referencia generales, de carácter selectivo y crítico. Abarca todas las áreas del conocimiento organizadas según una clasificación de materias propia y subdivididas por tipo de fuente (guías y manuales, bibliografías, enciclopedias,...). De alcance internacional, predominan los materiales en lengua inglesa. Basa la descripción de las fuentes en el soporte papel aunque indica si existe versión en soporte electrónico. Cuenta con índices de autor, título y materia. Existe una 11. ${ }^{a}$ ed. (1996) que se ha publicado también en CD-ROM. Incluye descripciones muy completas de las fuentes. Se actualiza con suplementos.

- GUIDE to reference books. Edit. by Robert Balay. 11.a ed. Chicago: American Library Association, 1996

Dividido en grandes grupos: obras generales, Humanidades, Ciencias Sociales, etc. Cuenta con índices de autores, títulos, materias. Aunque ofrece información de carácter internacional se advierte cierto sesgo a favor de Estados Unidos.

\section{- INDEX Bibliographicus. 4. ${ }^{a}$ ed. La Haye: FID, 1959-1964}

Recoge información sobre bibliografías en curso, generales o especializadas, en publicación independiente o incluidas en publicaciones periódicas. Ordenado por la CDU, se completa con índices por países. El volumen 2 se dedica a las Ciencias Sociales.

- La INFORMACIÓN especializada en Internet: directorio de recursos de interés académico y profesional. Coordinación Ángeles Maldonado Martínez y Luis Rodríguez Yunta. 2. ${ }^{a}$ ed. corr. y aum. Madrid: CSIC, 2006

Completa guía de fuentes de interés científico general que no sólo recoge las direcciones web, sino que también incluye en cada capítulo una introducción conceptual, así como breves descripciones de cada 
uno de los recursos. Se complementa con un directorio electrónico organizado por capítulos con todas las direcciones web que aparecen en el texto escrito.

- INTERNET invisible (http://www.internetinvisible.com)

Directorio internacional de bases de datos, de uso gratuito, al que se puede acceder por materias o por tipo de fuente.

- KATZ, William Armstrong: Introduction to reference work. 6 . $^{\mathrm{a}}$ ed. New York: McGraw-Hill, 1992. 2v.

Clásico manual sobre la referencia que incluye fuentes básicas organizadas por tipos. Centrado en la producción norteamericana y en parte inglesa, apenas se ocupa de otras fuentes extranjeras.

- MALCLÈS, Louise Nöelle: Les sources du travail bibliographique. 2ème.é. Génève: Droz, 1950-1958. 4 v.

Clásico manual francés de investigación bibliográfica y de consulta recomendable. Se convierte en una guía de gran valor para conocer las más importantes bibliografías de la historia. Los cuatro volúmenes están agrupados en tres tomos: I. Bibliografías generales II. Bibliografías especializadas: Ciencias Humanas y Sociales III. Bibliografías especializadas: Ciencias exactas y técnicas. La clasificación utilizada es sistemática.

- MALCLÈS, Louise Nöelle: Manuel de bibliographie. 4ème. éd. revue et mise à jour par Andrée Lhéritier. Paris: Presses Universitaires de France, 1986

Complemento del anterior y de fácil manejo. Recoge bibliografías generales y especializadas clasificadas por grandes áreas. Con índices de autores, títulos, obras anónimas y de materias.

- MARTÍN VEGA, Arturo: Fuentes de información general. Gijón: Trea, 1995

Manual básico de fuentes de información que resulta muy útil para iniciarse en las fuentes más destacadas.

- OBRAS de referencia electrónicas (http://wzar.unizar.es/doc/ buz/referencia.html)

Recurso de la biblioteca de la Universidad de Zaragoza que incluye información de diccionarios (monolingües, plurilingües, especializados), recursos en Internet, enciclopedias y otros. 
- SABOR, Josefa Emilia: Manual de fuentes de información. 3. ${ }^{a}$ ed. corr. y aum. Buenos Aires: Marymar, 1978

Referencia clásica en lengua española. Organiza las fuentes por tipos y es de cobertura internacional, aunque las referencias al mundo hispano son frecuentes. El problema es su deficiencia en cuanto a actualización. Las fuentes son de carácter general.

- SCHNEIDER, George: Handbuch der Bibliographie. $4 .^{\mathrm{a}}$ ed. Leipzig: K.W. Hiersemannn, 1930

Clásico de la Bibliografía alemana que incluye bibliografías generales anotadas.

- Les SERVICES bibliographiques dans le monde. 1950- . Paris: UNESCO, 1955- .

Obra apoyada por la UNESCO desde 1955. Se actualiza mediante listas bibliográficas que aparecen en el Boletín del Unisist del Programa General de Información de la UNESCO.

- SIMÓN DÍAZ, José: Bibliografía de la Literatura Hispánica. Volumen II. 2. ${ }^{\mathrm{a}}$ ed. Madrid: Instituto Miguel de Cervantes, C.S.I.C., 1962

La bibliografía de bibliografías española más completa que recoge bibliografías españolas y extranjeras que se refieren a España. Cada capítulo cuenta con un tipo de ordenación.

- TOOMEY, Alice F.: A world bibliography of bibliographies, 1964-1974: a decennal supplement to Theodore Besterman, A world bibliography of bibliographies. Totowa (New Jersey): Rowman and Littlefield, 1977. 2 v.

Realizada con los repertorios ingresados en la Biblioteca del Congreso de Washington.

- TOTOK, Wilhelm; Weitzel, Rolf: Handbuch der bibliographischen Nachschlagewerke. $4 .^{a}$ ed. Frankfurt am Main: Klostermann, 1954

Existe una edición italiana (Manuale internazionale di Bibliografia. A cura de Piero Innocenti. Milan: Editrice Bibliografica, 1979-1980, 4 v) que resulta más completa que la última edición alemana $\left(6 .^{\mathrm{a}} \mathrm{ed}\right.$. 1984-85) por aumentar la contribución bibliográfica de los países latinos. Organizada por tipos de fuentes, dedica unos capítulos a repertorios especiales. Se especializa en publicaciones en lengua alemana. 


\section{- UNIVERSIA (http://www1.universia.net)}

Es la mayor red universitaria de habla hispano-portuguesa en el mundo. Creada en el año 2000 , cuenta hoy con 70 universidades españolas y 840 americanas. Ofrece un gran número de recursos.

- UNIVERSIDAD Autónoma de Barcelona. Biblioteca. Recursos temáticos

(http://www.uab.es/servlet/Satellite?cid=1101231878128\&pagenam $\mathrm{e}=\mathrm{BibUAB} \% 2 \mathrm{FPage} \% 2 \mathrm{FTemplatePlanaBibUAB}$ )

- UNIVERSIDAD Autónoma de Madrid. Biblioteca. Recursos en Internet por Áreas

(http://biblioteca.uam.es/paginas/Recursos/recursos.html) terias

- UNIVERSIDAD Camilo José Cela. Biblioteca. Internet por ma(http://www.ucjc.edu/biblioteca/index.htm) ticas

- UNIVERSIDAD Carlos III de Madrid. Biblioteca. Guías temá-

(http://www.uc3m.es/uc3m/serv/BIB/GRAL/guias-recursos.html)

- UNIVERSIDAD Complutense de Madrid. Biblioteca. Internet por materias

(http://www.ucm.es/BUCM/frames07.htm)

- UNIVERSIDAD de Alcalá de Henares. Biblioteca

(http://www.uah.es/servi/biblioteca)

- UNIVERSIDAD de Alicante. Biblioteca. Servicio de información bibliográfica y documental

(http://www.ua.es/es/bibliotecas/SIBID/index.htm)

- UNIVERSIDAD de Almería. Biblioteca. Fuentes de información en Internet

(http://www.ual.es/Universidad/Biblioteca/internet/indice.htm)

- UNIVERSIDAD de Barcelona. Biblioteca. Guías temáticas

(http://www.bib.ub.edu/recursos-informacio/guies-tematiques) ción

- UNIVERSIDAD de Burgos. Biblioteca. Recursos de informa- 
(http://www.ubu.es/biblioteca/recursos_inet/8_2.htm)

- UNIVERSIDAD de Cádiz. Biblioteca. Recursos temáticos (http://biblioteca.uca.es) cursos.

- UNIVERSIDAD de Cantabria. Biblioteca. Áreas temáticas. Re(http://pc41.buc.unican.es/BDigital/areas/areas.htm)

- UNIVERSIDAD de Castilla-La Mancha. Biblioteca (http://www.biblioteca.uclm.es)

- UNIVERSIDAD de Córdoba. Biblioteca. Recursos electróni$\cos$

(http://www.uco.es/webuco/buc/?go=pages/recursos/recindex.html)

- UNIVERSIDAD de Extremadura. Biblioteca

(http://biblioteca.unex.es)

- UNIVERSIDAD de Girona. Biblioteca

(http://biblioteca.udg.es)

- UNIVERSIDAD de Granada. Biblioteca. Guías temáticas

(http://www.ugr.es/ biblio/biblioteca_electronica/guias_tematicas/ index.html)

- UNIVERSIDAD de Jaén. Biblioteca. Recursos electrónicos de Información científica htm)

(http://www.ujaen.es/serv/biblio/servicios/sad/bbdd/index_bbdd.

- UNIVERSIDAD de Huelva. Biblioteca. Biblioteca electrónica (http://www.uhu.es/biblioteca/index.htm)

- UNIVERSIDAD de La Coruña. Biblioteca. Recursos en Internet (http://www.udc.es/biblioteca/galego/index.htm) $\cos$

- UNIVERSIDAD de La Laguna. Biblioteca. Recursos electróni-

(http://papyrus.bbtk.ull.es)

- UNIVERSIDAD de La Rioja. Biblioteca. REI. Recursos Electrónicos de Información (http://aps.unirioja.es/biblio/recursos?sub=1) 
- UNIVERSIDAD de las Islas Baleares. Biblioteca.

(http://www.uib.es/servei/biblioteca)

- UNIVERSIDAD de Las Palmas de Gran Canaria. Biblioteca. Recursos electrónicos

(http://biblioteca.ulpgc.es/?q=servicios_metalib)

- UNIVERSIDAD de León. Biblioteca. Recursos electrónicos (http://biblioteca.unileon.es/bcadigital.shtml)

- UNIVERSIDAD de Málaga. Biblioteca. Recursos en Internet (http://www.uma.es/Servicios/Biblioteca/accesosexternos.htm)

- UNIVERSIDAD de Navarra. Biblioteca. Fuentes de referencia por materias

(http://www.unav.es/biblioteca/guias/fuentesreferencia.html)

- UNIVERSIDAD de Oviedo. Biblioteca

(http://buo.uniovi.es)

- UNIVERSIDAD de Salamanca. Biblioteca. Recursos en Internet por materias

(http://sabus.usal.es/recursospormateriasprincipal.htm)

- UNIVERSIDAD de Santiago de Compostela. Biblioteca. Recursos por materias

(http://busc.usc.es/)

- UNIVERSIDAD de Sevilla. Biblioteca. Guías por materias (http://bib.us.es/ayuda_invest/guiasmaterias.asp)

- UNIVERSIDAD de Valencia. Servicio de biblioteca y documentación

(http://biblioteca.uv.es/index.php)

- UNIVERSIDAD de Valladolid. Biblioteca. Guías temáticas (http://almena.uva.es)

- UNIVERSIDAD de Vigo. Biblioteca. Referencia e información bibliográfica htm)

(http://webs.uvigo.es/servicios/biblioteca/referencia/index_refer. 
- UNIVERSIDAD de Zaragoza. Biblioteca. Recursos Internet por materias

(http://wzar.unizar.es/doc/buz/materias.html)

- UNIVERSIDAD del País Vasco. Biblioteca. Recursos temáticos en Internet

(http://www.biblioteca.ehu.es/internet.htm)

- UNIVERSIDAD Jaume I. Biblioteca. Recursos en Internet (http://www.uji.es/cd/recursos/)

- UNIVERSIDAD Nacional de Educación a Distancia. Biblioteca. Recursos en Internet.

( http://portal.uned.es/portal/page?_pageid= 93,511995\&_dad=portal\&_schema=PORTAL)

- UNIVERSIDAD Pompeu Fabra. Biblioteca. Guías temáticas (http://www.upf.es/bib/)

- UNIVERSIDAD Pontificia de Comillas. Biblioteca. Fuentes de información 15)

(http://www.upcomillas.es/servicios/serv_bibl.aspx?nivel=2\&inicio=

- UNIVERSIDAD Pontificia de Salamanca. Biblioteca

(http://www.biblioteca.upsa.es)

- UNIVERSIDAD Ramón Llull. Biblioteca

(http://www.url.es/cont/url/biblioteques.php)

- UNIVERSIDAD Rey Juan Carlos. Biblioteca. Recursos temáti$\cos$ htm)

(http://www.urjc.es/z_files/ac_biblio/nuevaweb/tematicos/tematicos.

- UNIVERSIDAD Rovira i Virgili. Biblioteca

(http://www.urv.es/biblioteca/index.html)

- WALFORD`s guide to reference materials. 7th. ed. London: Library Association, 1996-1998. 3 v.

Guía selectiva y crítica de obras de referencia de carácter general. De alcance internacional, predominan las referencias británicas y 
europeas. Abarca todas las materias e incluye fuentes en cualquier formato con unas descripciones muy completas. Ordena las materias por la CDU y las fuentes, dentro de cada materia, por tipo (bibliografías, enciclopedias...). Da preferencia a las fuentes actuales y en curso, informa de bibliografías incluidas en publicaciones periódicas o en libros e indica la disponibilidad de la fuente en soporte informático. De cuidada actualización.

\section{II.2. GUIAS ESPECIALIZADAS EN HUMANIDADES Y CIENCIAS SOCIALES}

Destacamos las que tienen que ver con la materia objeto de estudio:

- BARBERENA BLÁZQUEZ, Elsa; Noguez Ortiz, Araceli: Las Humanidades en web y en CD-ROM. México: UNAM, 1999

Ofrece una relación selectiva de las páginas web y bases de datos en disco compacto y en línea especializados en Humanidades. Cuenta con índice de proveedores. Cada registro posee información sobre el contenido, la cobertura temática, el idioma, la cobertura geográfica, la institución responsable, la edición, el acceso, el proveedor y el precio de cada uno de los recursos.

- BLAZEK, Ron; Aversa, Elizabeth: The Humanities: a selective guide to information sources. 4th. ed. Englewood: Libraries Unlimited, 1994

Guía selectiva y crítica, de carácter internacional, que se organiza en 12 capítulos dedicados los pares (fuentes) a describir las fuentes y los impares (de acceso) a aspectos teórico-conceptuales de las humanidades en general, y de cada una de las disciplinas estudiadas. Los asientos están anotados y se ordenan por tipo de fuente. Las descripciones son muy completas. Contiene un índice de autores y otro temático. Requiere ser actualizado.

- CARRIZO SAINERO, Gloria: La información en Ciencias Sociales. Gijón: Trea, 2000

Aproximación básica al concepto de Información y Documentación en Ciencias Sociales con referencias de fuentes organizadas por disciplinas y tipos. 
- GARZA MERCADO, Ario: Fuentes de información en Ciencias Sociales y Humanidades. México: El Colegio de México, Biblioteca Daniel Cosío Villegas, 1999- . 3v.

Guía de alcance internacional aunque con énfasis en las fuentes mexicanas y latinoamericanas. Recoge obras de referencia que se incluyen en la Biblioteca Daniel Cosío Villegas. Incluye fuentes de especial interés para estudios sobre el siglo XX en español, francés e inglés. Las entradas están anotadas. El primer volumen está organizado por materias, dedicando un capítulo a obras de carácter general. Cada capítulo dedicado a distintas disciplinas está ordenado por tipo de fuente. Ofrece la signatura topográfica de la biblioteca a la que pertenecen.

- GARZA MERCADO, Ario: Obras de consulta para estudiantes de Ciencias Sociales y Humanidades. México: El Colegio de México; Biblioteca Daniel Cosío Villegas; 1992

De idénticas características que el anterior, aunque ordenado por tipos de fuentes y con una intención más generalizadora.

- The HUMANITIES and the library. Edited by Nena Couch and Nancy Allen. 2nd. ed. Chicago: American Library Association, 1993

Organizada por capítulos dedicados a cada una de las disciplinas de las Humanidades, se ocupa más de fuentes en lengua inglesa y ofrece descripciones que no siempre son completas. Cuenta con índices de autores y materias. Complemento de la de Blazek por incorporar información más actualizada y distintos tipos de formatos.

- LI, Tze-Chung: Social science reference sources: a practical guide. 3ed. ed. Westport (Connecticut), London: Greenwood Press, 2000

Guía básica dividida en dos partes: Ciencias sociales en general y algunas disciplinas en particular. Contiene completas descripciones valorativas. No solo incluye fuentes, sino también reflexiones sobre la Información y Documentación en estas Ciencias.

- ROMANOS DE TIRATEL, Susana: Guía de fuentes de información especializadas: Humanidades y Ciencias Sociales. Buenos Aires: EB publicaciones, 1996

Ofrece información conceptual sobre la información y las fuentes de información especializadas en Humanidades y Ciencias Sociales así como una breve, pero completa guía, de las más significativas a 
nivel internacional con asientos valorativos de cada una de ellas. Hace breves introducciones a cada uno de los tipos.

- ROMANOS DE TIRATEL, Susana: Guía de fuentes de información especializadas: Humanidades y Ciencias Sociales. 2. ${ }^{a}$ ed. Buenos Aires: GREBYD, 2000

La única novedad respecto a la anterior edición es la supresión de un capítulo dedicado a las bibliotecas especializadas, la inclusión de algunos repertorios y la ampliación de la sección dedicada a las guías de obras de referencia especializadas.

- ROSENFELD, Louis B.; Janes, Joseph; Vander Kolk, Martha: The Internet compendium: subject guides to humanities resources. New York: Mansell, 1995

Contiene dieciséis capítulos dedicados a recursos de distintas disciplinas y un índice de materias.

- ROSENFELD, Louis B; Janes, Joseph; Vander Kolk, Martha: The Internet compendium: subject guides to social sciences, business and law resources. New York: Mansell, 1995

De idénticas características que el anterior.

- The SOCIAL Sciences: a cross-disciplinary guide to selected sources; general editor Nancy L. Herron. 2nd. ed. Englewood (Colorado): Libraries Unlimited, 1996

Describe minuciosamente las fuentes seleccionadas, agrupando sus capítulos en cuatro partes: fuentes generales de las Ciencias Sociales, literatura de las disciplinas consolidadas (Ciencia Política y Derecho, entre otras), literatura de las disciplinas emergentes y literatura de las disciplinas relacionadas. Incluye índices de autores, títulos y materias. En esta segunda edición, se han ampliado los ensayos introductorios y las secciones sobre recursos electrónicos.

- WEBB, William H.: Sources of information in the Social Sciences: a guide to the literature. 3rd. ed. Chicago: American Library Association, 1986

De alcance internacional, cubre el campo de las Ciencias Sociales en general y de algunas disciplinas en particular, como son Historia y Ciencia política entre otras. Organizada en capítulos que incluyen un estudio de las respectivas materias y una lista anotada de obras de referencia ordenadas por tipo de fuente. Contiene índices de autores, títulos y materias. 


\section{II.3. GUÍAS ESPECIALIZADAS EN FILOSOFÍA}

- ANÁBASIS digital. Portal bibliográfico de Filosofía (http://www.anabasisdigital.com)

Nueva etapa digital de una revista de información bibliográfica. Un esfuerzo pionero en el ámbito de la Filosofía en lengua española. Novedades y enlaces a las editoriales.

- BYNAGLE, Hans E.: Philosophy: a guide to the reference literature. 2nd. ed. Englewood (Colorado): Libraries Unlimited, 1997.

Amplia en cobertura, actualizada y anotada. Aunque presta más atención a las fuentes en inglés, no olvida otras en lenguas europeas. El primer capítulo es introductorio y el resto se ordena por tipos de obras de referencia. Incluye un capítulo dedicado a recursos electrónicos y otros dos a revistas y organizaciones y centros de investigación. Tiene índices de autor, título y materia. Red

- CENTRO Telemático de Filosofía. Recursos filosóficos en la

(http://www.blues.uab.es/filosofia/centrotelematico.html)

Colección de recursos informáticos para la Filosofía a cargo de Jesús Hernández Reynés y el departamento de Filosofía de la Universidad Autónoma de Barcelona. Se estructura en 4 apartados: Recursos (buscadores especializados en Filosofía, webs generales y temáticas, filósofos y escuelas, páginas personales y guías), Publicaciones (textos electrónicos, monografías, revistas, enciclopedias, bases de datos, directorios de textos electrónicos), Libros (catálogos generales, bibliotecas, librerías, editoriales, bibliografías, reseñas) e Instituciones (departamentos y centros universitarios, asociaciones y centros, institutos y cátedras, grupos de investigación, organismos oficiales.

- FILOSOFÍA. NET. El portal de la Filosofía en Internet (http://www.filosofia.net)

Incluye portales, buscadores, diccionarios, manuales, foros de discusión, sociedades, revistas e instituciones de Filosofía.

- IZUZQUIZA, Ignacio: Guía para el estudio de la Filosofía. Referencias y métodos. Barcelona: Anthropos; Santafé de Bogotá: Siglo del Hombre, 1994

Viene a llenar un vacío ofreciendo un conjunto de indicaciones metodológicas y una serie de referencias introductorias para el estudio y la investigación en Filosofía y en castellano. Resulta algo des- 
fasado por no recoger la incidencia de los procesos informáticos en relación con la Documentación. Ordena las fuentes por tipos y recoge tanto documentales como institucionales. Las referencias son anotadas. Incluye bibliografías de algunos filósofos importantes, de diferentes disciplinas filosóficas, una relación de publicaciones periódicas de interés en el campo de la Filosofía en general.

- LECHUZA, la documentación filosófica en español. (http://www.lechuza.org)

Servicio integral al servicio de la documentación. Localiza documentos, sitios de Internet, etc..., de interés filosófico. Los fondos están en la Biblioteca de la Fundación Gustavo Bueno.

- MUÑOZ-ALONSO LÓPEZ, Gemma: "Las Fuentes de información documentales en el ámbito de la Filosofía», en Métodos didácticos en Biblioteconomía y Documentación. VI Jornadas Académicas organizadas por la EUBD de la UCM. Madrid: UCM, 1997, pp. 355-371

Tras una breve introducción sobre el significado de las fuentes de información, recoge algunas de las más significativas organizándolas según la clasificación de fuentes documentales primarias y secundarias. En el primer bloque incluye «historias de la Filosofía» escritas o traducidas al español, y en el segundo algunos ejemplos de obras de consulta y fuentes de información bibliográfica.

- MUÑOZ ALONSO LÓPEZ, Gemma: "Información on line en Filosofía», en Revista General de Información y Documentación, vol. 7 (2), 1997, pp. 375-383

Breve pero completa información de algunos sitios web relacionados con el campo de la Filosofía y ordenados alfabéticamente.

- MUÑOZ ALONSO LÓPEZ, Gemma: «Uso y disfrute de enciclopedias y diccionarios", en Anales del Seminario de Historia de la Filosofía, 1998, (15), pp. 101-130

La autora presenta distintos criterios de evaluación aplicables a enciclopedias y diccionarios, así como una selección de algunos especializados en Filosofía, con comentarios sobre su contenido y adecuación.

- PROYECTO Filosofía en español. (http://www.filosofia.org)

Estructura que impulsa la creación, mantenimiento y difusión de diversos recursos documentales, ediciones digitales o electrónicas y otros mecanismos que facilitan la comunicación, con vocación de in- 
corporar cuanto tenga que ver con la Filosofía escrita en lengua española. Existe desde 1995. Fue iniciativa de la revista El Basilisco, pero al integrarse ésta en 1998 en la Fundación Gustavo Bueno, fue esta institución la que asumió completamente el proyecto. Incluye ediciones digitales de textos de autores en lengua española, foros de discusión, enlaces a revistas y a artículos desde el siglo XIX (texto completo), información sobre convocatorias y sociedades y novedades bibliográficas e información biográfica.

- UNIVERSIDAD Autónoma de Madrid. Biblioteca de Humanidades. Recursos de Filosofía en Internet (http://biblioteca.uam.es/ paginas/Humanidades/enl_filosofia.html)

- UNIVERSIDAD Complutense de Madrid. Biblioteca. Facultad de Filosofía. Filosofía en Internet (http://www.ucm.es/ BUCM/fsl/0500.htm)

- UNIVERSIDAD de Alicante. Biblioteca de Filosofía y Letras. Recursos por materias htm)

(http://www.ua.es/es/bibliotecas/SIBID/centros/neo_letras/rec_mat.

- UNIVERSIDAD de Cantabria. Biblioteca. Recursos por áreas. Filosofía

(http://pc41.buc.unican.es/BDigital/areas/cadaarea.asp?materia= Filosofía\&centro=INT)

- UNIVERSIDAD de Granada. Biblioteca. Guías temática. Filosofía y Religión

(http://www.ugr.es/ bibliofl)

- UNIVERSIDAD de La Laguna. Biblioteca. Recursos electrónicos. Filosofía

(http://absysweb.ccti.ull.es/cgi-bin/abwebp.exe/L1/T50/G0?ACC= DIR\&EXP=Filosofía.NULL.) losofía

- UNIVERSIDAD de las Islas Baleares. Biblioteca. Novedades Fi-

(http://www.uib.es/servei/biblioteca/info/novetats/novetats_ bibliografiques.htm) Filosofía 
(http://www.uma.es/servicios/biblioteca/RecursosHumanidades. htm\#FILOSOFIA)

- UNIVERSIDAD de Navarra. Biblioteca. Fuentes de referencia por materias. Filosofía

(http://www.unav.es/biblioteca/guias/referenciafilosofia.html)

- UNIVERSIDAD de Salamanca. Biblioteca. Recursos en Internet por materias. Filosofía (http://sabus.usal.es/filosofia.htm)

- UNIVERSIDAD de Santiago de Compostela. Biblioteca. Recursos por materias. Filosofía

(http://busc.usc.es/Recursos_en_Internet/Recursos_por_materias/ rec_filosofia.asp)

- UNIVERSIDAD de Sevilla. Biblioteca de Psicología y Filosofía. Recursos en Filosofía

(http://www.aloj.us.es/bibpsico/recursosfilosofia.htm)

- UNIVERSIDAD de Valladolid. Biblioteca. Guías temáticas. Humanidades

(http://www.uva.es/uva/export/portal/com/bin/contenidos/servicios Administrativos/bibliotecaArchivo/Biblioteca_universitaria/Guias_ Tematica/1175070761061_guxa_de_recursos_de_humanidades webmarzo2007.htm)

- UNIVERSIDAD Ramón Llull. Biblioteca de Filosofía (Biblioteca Pública Episcopal del Seminario de Barcelona) (http://www.bibliotecaepiscopalbcn.org)

- UNIVERSIDAD Nacional de Educación a Distancia. Biblioteca. Recursos en Internet. Filosofía (http://www.uned.es/biblioteca/ recursos/nv/filosofia.htm)

\section{II.4. GUÍAS ESPECIALIZADAS EN DERECHO Y FILOSOFÍA JURÍDICA}

- DERECHO.COM (http://www.derecho.com)

Portal que ofrece contenidos y servicios legales de calidad a través de Internet.

- DERECHO y Estado en Internet: guía básica de direcciones. Madrid, etc: Prentice Hall, 2001 
Pequeña guía que ofrece direcciones de instituciones, asociaciones y otros sitios de interés con una breve, pero práctica, descripción de algunas de ellas. El libro está estructurado en 9 capítulos: Altas instituciones del Estado, Administración general del Estado, Administración territorial, local, colegios profesionales, universidades, instituciones internacionales, becas y ofertas de empleo y sitios de interés. A destacar el capítulo dedicado a las universidades, españolas y públicas y privadas, por ser instituciones de gran valor debido a sus bibliotecas, archivos y centros de documentación.

- DERECHO público en Internet: guía de direcciones. Ed. Por Gerardo Ruiz-Rico Ruiz y Juan José Ruiz Ruiz. Madrid: Centro de Estudios Políticos y Constitucionales, 2003

Recoge un gran número de direcciones de interés para el investigador en Derecho público organizadas según el criterio que siguen los habituales manuales de Derecho constitucional. Incluye información de editoriales, centros de documentación, boletines oficiales, revistas electrónicas, foros de debate, etc. De interés por la información que aporta sobre derechos humanos.

\section{- Derechos HUMAN RIGHTS}

\section{(http://www.derechos.org)(http://www.derechos.net/links/esp)}

Organización internacional nacida en Internet que ofrece información sobre novedades, publicaciones (artículos, monografías, bases de datos, bibliografías, boletines de ONGS, revistas académicas) y enlaces de derechos humanos (acciones y campañas, legislación y jurisprudencia, directorios de enlaces, foros de discusión, informes y noticias, organismos internacionales, ONGs...).

- DOXA. Filosofía del Derecho (http://www.cervantesvirtual.com/ portal/DOXA)

Trata de ofrecer en soporte digital un conjunto amplio de servicios de documentación de interés para la comunidad de filósofos del Derecho que se expresan en lengua española. Entre sus enlaces ofrece información sobre revistas, catálogos de bibliotecas, tesis doctorales y publicaciones electrónicas. com)

- FILOSOFÍAYDERECHO.com (http://www.filosofiayderecho.

Incluye como sitios de Filosofía del Derecho: Revista Telemática de Filosofía del Derecho (desde 1997, sólo en Internet y gratuita), Grupo de investigación en Teoría general del Derecho, Enlaces a 
otros sitios de Filosofía jurídica (asociaciones, departamentos y centros de España, Europa e Iberoamérica, Revistas, Editoriales y páginas personales). Permite alojar las web de departamentos, institutos de investigación, grupos de trabajo, etc... que lo deseen. Cuenta con una lista de distribución a la que se puede suscribir el usuario. Además de los sitios, presenta otro bloque de Novedades. Otros puntos de interés son la Biblioteca-e (relación de pensadores con sus obras y bio-bibliografías) y Agenda (información de Congresos).

- HERNÁNDEZ APARICIO, Mercedes: Guía del servicio de información y referencia de Derecho de la UCM. Madrid: Biblioteca UCM, 1995

Se centra en repertorios de legislación y jurisprudencia, en papel y soporte informático. Incluye también algunos diccionarios especializados.

- INFODERECHO (http://www.infoderecho.com)

Portal jurídico dedicado a los sitios jurídicos más novedosos y populares de la red.

- IUSTEL, Portal del Derecho (http://www.iustel.com)

Incluye distintos servicios. En el apartado Biblioteca recoge una base de datos referencial de libros y artículos, algunos digitalizados. También contiene revistas electrónicas de interés.

- JURISWEB: Derecho español en Internet (http://www.jurisweb.com)

Organizado de forma sistemática. Desde sus páginas se puede acceder a textos legales, jurisprudenciales, instituciones, boletines oficiales, departamentos universitarios...

- MANUAL de documentación jurídica. Edit. por Mateo Maciá. Madrid: Síntesis, 1998

Más que una guía de fuentes propiamente dicha, se trata de un manual de introducción al complejo mundo de la denominada Documentación jurídica. Se abordan algunos temas relacionados con este asunto tales como tipología y caracterización, documentaciones legislativa, judicial, de doctrina jurídica o parlamentaria y la Documentación jurídica en su relación con Internet.

- NOTICIAS jurídicas (http://noticias.juridicas.com)

Ofrece novedades en legislación y jurisprudencia, artículos doctrinales y boletines a texto completo. 
- PÁEZ MAÑÁ, Jorge: Bases de datos jurídicos. Madrid: CINDOC, 1994

Junto a un completo estudio teórico sobre la Documentación jurídica y las bases de datos jurídicos, incluye un repertorio de bases de datos jurídicos españolas, comunitarias, francesas, italianas, inglesas, alemanas, belgas y estadounidenses, indicando datos tales como nombre, productor, distribuidor, soporte y sistema de recuperación, actualización, temática, cobertura cronológica, tipología documental que incluye, estructura de la información y volumen de documentos.

- PÓRTICO legal.com (http://www.porticolegal.com)

Cuenta con más de 8000 enlaces relacionados con el mundo del Derecho. goza

- SERVIDOR de derechos humanos de la Universidad de Zara-

(http://www.unizar.es/derechos_humanos)

Ofrece información sobre programas de doctorado, recursos de Internet, bibliografía, convocatorias de encuentros, jornadas, cursos, etc..., relacionados con los derechos humanos.

- TODALALEY.com (http://www.todalaley.com)

Portal que recoge la legislación actual, tanto estatal como autonómica, formularios administrativos y procesales, modelos de contratos y guías civiles, mercantiles y laborales, y que permite su consulta de forma totalmente gratuita, aunque algunos de sus contenidos, como son los boletines oficiales, requieren de un abono para ello.

- UNIVERSIA. Aula digital. Recursos jurídicos comentados (http://estudios.universia.es/recursos/auladigital/derecho)

- UNIVERSIDAD Alfonso X el Sabio. Biblioteca. Recursos (http://www.uax.es/biblioteca/recursos_frame.html)

- UNIVERSIDAD Autónoma de Madrid. Facultad de Derecho. Biblioteca. Selección de Recursos Jurídicos en Internet

(http://biblioteca.uam.es/paginas/Derecho/rec_internet.html)

- UNIVERSIDAD Carlos III de Madrid. Biblioteca. Recursos de Información en Derecho (http://www.uc3m.es/uc3m/serv/BIB/ GUIA/derecho.html) 
- UNIVERSIDAD Complutense de Madrid. Facultad de Derecho. Derecho en Internet

(http://www.ucm.es/BUCM/der/0500.htm)

- UNIVERSIDAD de Alcalá de Henares. Facultad de Derecho. Biblioteca. Recursos jurídicos (http://www.uah.es/derecho/servicios/ biblioteca.htm)

- UNIVERSIDAD de Alicante. Facultad de Derecho. Biblioteca. Recursos por materias htm)

(http://www.ua.es/es/bibliotecas/SIBID/centros/neo_derecho/index. ción

- UNIVERSIDAD de Almería. Biblioteca. Recursos en Legisla-

(http://www.ual.es/Universidad/Biblioteca/legislacion/marcoleg2. htm)

- UNIVERSIDAD de Barcelona. Biblioteca. Recursos en Derecho (http://www.bib.ub.es/www2/2intdret.htm)

- UNIVERSIDAD de Burgos. Biblioteca. Recursos en Internet. Recursos por Materias. Derecho (http://www.ubu.es/biblioteca/ recursos_inet/8_2_m.htm)

- UNIVERSIDAD de Cádiz. Biblioteca. Recursos Jurídicos (http://biblioteca.uca.es/sbuca/bibcjer/recujur.asp)

- UNIVERSIDAD de Cantabria. Biblioteca. Biblioteca. Recursos por áreas. Derecho

(http://pc41.buc.unican.es/BDigital/areas/cadaarea.asp?materia=De recho\&centro $=\mathrm{DEC}$ )

- UNIVERSIDAD de Castilla - La Mancha. Departamento de Derecho internacional Privado (http://estudios.universia.es/recursos/ auladigital/derecho/uclm/)

- UNIVERSIDAD de Córdoba. Facultad de Derecho. Biblioteca. Recursos electrónicos (http://www.uco.es/webuco/buc/centros/der) con

- UNIVERSIDAD de Extremadura. Facultad de Derecho. Juridi-

(http://www-derecho.unex.es/biblioteca/juridicon.htm) 
- UNIVERSIDAD de Girona. Departamento de Derecho civil Página Jurídica = Spanish Legal Page (http://civil.udg.es/pagina) web

- UNIVERSIDAD de Granada. Facultad de Derecho. Recursos

(http://www.ugr.es/\%7Ewderecho/recursos_internet.htm)

UNIVERSIDAD de Murcia. Facultad de Derecho. Biblioteca. Enlaces de interés

(http://www.um.es/biblio/BCA/general/derecho.htm)

- UNIVERSIDAD de Oviedo - Derecons (http://constitucion. rediris.es/Princip.html)

- UNIVERSIDAD de Salamanca. Biblioteca. Recursos Internet. Derecho

(http://sabus.usal.es/derecho.htm)

- UNIVERSIDAD de Santiago de Compostela. Facultad de Derecho. Recursos jurídicos en Internet (http://www.usc.es/ fac_dereito/recursosxuridicos.htm)

- UNIVERSIDAD de Sevilla. Biblioteca. Guía por Materias

(http://bib.us.es/guiaspormaterias/ayuda_invest/derecho/guiaspor materias01.htm) en Internet

- UNIVERSIDAD de Valencia. Biblioteca. Recursos Jurídicos (http://www.uv.es/biblios/webs/juridicos.html)

- UNIVERSIDAD de Vigo. Facultad de Derecho. Enlaces (http://dereito.uvigo.es/\#enlaces)

- UNIVERSIDAD de Zaragoza. Facultad de Derecho. Biblioteca. Información jurídica en Internet (http://biblioteca.unizar.es/ archivos_biblio/recursos/Recursframe.html) de interés

(http://www.zuzenbide.ehu.es/p045-645/es/contenidos/enlace/ fderecho_enlaces_interes/es_enlaces/enlaces_derecho.html)

- UNIVERSIDAD Nacional de Educación a Distancia. Facultad de Derecho. Enlaces de interés jurídico 
(http://portal.uned.es/portal/page?_pageid=93,161966\&_dad= portal\&_schema=PORTAL)

- UNIVERSIDAD Pompeu Fabra. Biblioteca. Guía de Información sobre Derecho español (http://www.upf.edu/bib/dret)

- UNIVERSIDAD Rovira i Virgili. Biblioteca. Recursos electrónicos seleccionados. Ciencias jurídicas y sociales (http://wwwa.urv.net/biblioteca/relec)

- Vlex (http://www.vlex.com)

VLex es una editorial jurídica que ofrece sus productos y servicios en Internet. Su principal ventaja ante las demás editoriales jurídicas es que aprovecha la inmediatez que les ofrece el medio y proporciona la información actualizada al día. 